\title{
MOLEKULARES DESIGN FÜR INNOVATIVE PU-SYSTEME
}

\author{
Vesela Stoycheva, Harald Goering, Uta Knoll
}

\section{Zusammenfassung}

Da Polyurethane (PU) mithilfe einer Polyadditionsreaktion gebildet werden, ist diese Stoffklasse besonders geeignet, durch den Einsatz neuartiger Komponenten, Materialien mit innovativen Eigenschaftsspektren zu synthetisieren. So wird es möglich, für elektrische und elektronische Module, Verguss- und Einbettmassen mit den erforderlichen mechanischen Eigenschaften bei guter Verarbeitbarkeit herzustellen.

Durch neuartige Synthesewege werden hierzu besonders hydrophobe Polyole auf Basis von 2-Ethylhexan-1,3-diol und Terephthalsäure entwickelt, die einen großen Anteil an nachwachsenden Rohstoffen in Form von nativem Öl enthalten. Für den praktischen Einsatz als 2-Komponenten-Vergussmasse muss jedoch aus verarbeitungstechnischen Gründen die Viskosität des entwickelten oligomeren Diols deutlich erniedrigt werden. Dazu dienen die eingesetzten pflanzlichen Öle wie Raps- oder Sojaöl. Da für die Eigenschaften nicht nur der chemische Aufbau der Molekülketten, sondern auch die Morphologie des PU Systems entscheidend sind, wird mithilfe thermo analytischer Messmethoden und abbildender Verfahren die bei dem neuartigen Syntheseweg ausgebildete Phasenstruktur charakterisiert.

\section{Abstract}

Polyurethanes (PU) are formed by an addition reaction, therefore they are suited for modifications by further components to synthesize materials with sophisticated properties. By this way it is possible to produce sealing compounds for electrotechnical and electronical applications with excellent mechanical and processing properties.

Especially hydrophobic polyols, based on 2-ethylhexane-1,3diole and terephthalic acid were developed, which are able to enter a considerable amount of renewable ressources in terms of plant oils. The use in two-component sealant systems demands a notable reduction of the viscosity of the developed oligomeric diol. For his purpose rapeseed and soybean oils are used. The properties of polyurethanes are determined by both the chemical structure of the molecular chains and the morphology of the polymeric system. Hence thermoanalytic and imaging methods were used to investigate the phase structures formed during the new developed synthesis route.

\section{»I. EINFÜHRUNG}

Mit der hier dargestellten Entwicklung von neuen PU-Systemen wird auf niedrigviskose, katalysatorfreie Harzsysteme fokussiert, die aufgrund ihrer starken Hydrophobie als Vergussmassen in elektrotechnischen Anwendungen nachgefragt sind.

Durch die Synthese mikrophasenseparierter Multikomponentensysteme werden, auf einer Größenskala von Nanometern, Strukturen mit konträrem Verhalten miteinander vereint, um so Polyurethane (PU) mit besonderen Eigenschaften zu erhalten. Das gezielte molekulare Design setzt die detaillierte Kenntnis von relevanten StrukturEigenschaftsbeziehungen voraus. Diese werden an PU-Systemen eingehend untersucht, die im Rahmen des Gesamtvorhabens synthetisiert wurden. Dabei steht der Einfluss der ausgebildeten Morphologie der mithilfe der ZweiKomponenten-Technik hergestellten, schwach vernetzten PU-Systeme, auf deren elektrische Eigenschaften, besonders der elektrischen Durchschlagsfestigkeit, im Mittelpunkt des Interesses (Leppekes 2003).

Bei der Synthese der Polyurethan-Netzwerke mit den für eine Kabelvergussmasse geeigneten Eigenschaften wird die gegenseitige Beeinflussung von Polyadditionsreaktion und physikalischen Phasenseparationsprozessen zur Gestaltung der gewünschten Morphologie ausgenutzt (Stoycheva et al. 2010). Sowohl die Maschenweite des gebildeten Netzwerks als auch die Steifigkeit der Molekülsegmente sind für die Endeigenschaften maßgeblich.

Eine Größe zur Charakterisierung des Härtungszustands ist die Glasübergangstemperatur $T_{g}$. Je enger die Netzmaschen oder mit anderen Worten, je vollständiger die Reaktion der funktionellen Gruppen, umso höher liegt $T_{g}$. Übersteigt die Härtungstemperatur $T_{g}$, dann geht das Material vom „eingefrorenen“ starren Zustand in den "gummielastischen" flexiblen Zustand über. Die Härtungsreaktion kann weiterlaufen bis bei Erreichen der nun zu höheren Temperaturen verschobenen neuen Glastemperatur die Reaktion wiederum „einfriert“. Die maximal erreichbare Glastemperatur wird $T_{g} \infty$ genannt. Das Überschreiten von $T_{g} \infty$ bewirkt keine weitere Reaktion, da alle möglichen Verknüpfungen hergestellt sind.

Diese Effekte werden überlagert von der Aggregation einzelner Abschnitte der sich bildenden Makromoleküle. Die Zusammenlagerung von Kettensegmenten zu separaten Phasen wird vor allem durch besonders starke zwischenmolekulare Wechselwirkungen verursacht (Oertel 1993). Die Ausbildung solcher Phasen erfolgt während der PU-Reaktion und hängt deshalb auch in starkem Maß von der Viskosität (Beweglichkeit) der einzelnen Reaktanten ab (Randall et al. 2002). 


\section{» II. EXPERIMENTELLES}

Für die angestrebte deutliche Verlängerung der Verarbeitungszeit wurden neuartige Polyesteralkohole auf Basis von 2-Ethylhexan-1,3-diol (EHD) und Terephthalsäure (TPA) entwickelt (Stoycheva et al. 2008). Die Reaktion verläuft prinzipiell nach folgender Gleichung (Abb. 1):<smiles>CCCC(O)C(CC)CO</smiles>

EHD
TPA<smiles>O=C(O)c1ccc(C(=O)O)cc1</smiles><smiles>CCCC(O)C(CC)CO</smiles>

EHD
Der synthetisierte Diester hat eine erwartete mittlere Molmasse von ca. 450 $\mathrm{g} / \mathrm{mol}$ und eine $\mathrm{OH}-\mathrm{Zahl}$ von $310 \mathrm{mg}$ $\mathrm{KOH} / \mathrm{g}$ (überwiegend freie sekundäre $\mathrm{OH}$-Gruppen) (Ionescu 2005). Für den praktischen Einsatz als 2-KomponentenVergussmasse muss jedoch aus verarbeitungstechnischen Gründen die Viskosität des entwickelten oligomeren Diols deutlich erniedrigt werden. Dazu werden pflanzliche Öle wie Raps- oder Sojaöl verwendet. Diese lassen sich in weiten Grenzen mit den Diolen mischen und bilden stabile homogene DiolÖl-Mischungen mit bis zu 40 wt.-\% Raps- oder Sojaöl. Die Mischungen sind dauerhaft klar und homogen. Die Säure- und Hydroxylzahlen der Gemische sinken in den theoretisch zu erwartenden Bereich. Die Viskositäten der Diol-Öl-Gemische mit Raps- oder Sojaöl werden deutlich von ca. 10 Pas auf bis zu 1 Pas erniedrigt (Desroches et. al. 2012).

PU-Vergussmassen wurden aus den entwickelten Diolen bzw. Diol-ÖlGemischen und polymerem Diphenylmethandiisocyanat ( $p-M D I$, Lupranat M20S der BASF SE) hergestellt (Abb. 2). Die Synthese konnte ohne Katalysatoren und Hilfsstoffe realisiert werden. Die Synthese erfolgte bei $120^{\circ} \mathrm{C}$ über $3 \mathrm{~h}$ im Trockenschrank.

Zur Messung von $\mathrm{T}_{\mathrm{g}}$ gibt es eine Vielzahl von Messmethoden. Da diese Temperatur einen Übergangsbereich als physikalisches Phänomen beschreibt, muss zur Bewertung stets die Messmethode, einschließlich der jeweiligen Messparameter, angegeben werden. Eine der am häufigsten angewendeten experimentellen Methoden zur Bestimmung von $\mathrm{T}_{\mathrm{g}}$ ist die dynamische Differenzkalorimetrie (DSC). In einer DSC-Apparatur wird mit einem kleinen Ofen Wärme zur Probe und zu einer inerten Referenz übertragen. In unmittelbarem Kontakt zu Probe und Referenz sind Temperatursensoren (Thermoelemente) angebracht. Sie ermöglichen die Registrierung der Temperaturdifferenz zwischen Probe und Referenz während des zeitlinearen Aufheizens (Hatakeyma 1999). Dieses Signal dient als Maß für den Wärmefluss zwischen Probe und Referenz.

Ein exothermer Prozess zeigt einen positiven Peak, während endotherme 
Prozesse negative Peaks hervorrufen. Aus solchen Kurven kann die Enthalpie, d. h. die "Wärmetönung“ einer Umwandlung, berechnet werden (Höhne et al. 2003). Sie entspricht der integrierten Fläche des zur Umwandlung gehörigen Peaks. Die Glasumwandlung erscheint als Stufe in der Basislinie des registrierten DSC-Signals, da die Probe im Bereich der Glasumwandlung ihre Wärmekapazität ändert. Die Vernetzung von Kettenmolekülen während des Härtungsprozesses erzeugt als exothermer Vorgang einen positiven Peak in der DSC-Kurve. Abbildung 3 zeigt eine für einen Kunststoff typische DSC-Heizkurve.

\section{» III. ERGEBNISSE UND DISKUSSION}

Im Allgemeinen werden bei der Auswertung von DSC-Messungen die 2. Heizkurven verwendet, um durch Aufschmelzen beim 1. Heizlauf einen guten Wärmekontakt der Probe zum Messtiegel zu erreichen. Allerdings geht dann die thermische Vorgeschichte, die sowohl Auswirkungen auf die Morphologie als auch den Härtungszustand hat, verloren. Als Beispiel wird in Abbildung 4 der 1. und 2. Heizlauf für eine Modellprobe gezeigt. Wie der 1. Heizkurve zu entnehmen ist, lässt sich $T_{g}$ nur mit einer relativen Unsicherheit bestimmen. Die Schulter bei etwa $70^{\circ} \mathrm{C}$ entsteht durch die Überlagerung des Glasübergangs mit einem endothermen Peak (Verdampfen). Im 2. Heizlauf ist sie nicht mehr zu finden. Zum einem ist zu erkennen, dass die mit $\mathrm{T}_{\mathrm{g}}$ (höhere molekulare Beweglichkeit) einsetzende Nachrektion die Glastemperatur weiter zu höheren Temperaturen verschiebt. Bei noch höheren Temperaturen überwiegt dann zum anderen die auftretende Reaktionswärme den Glasumwandlungseffekt und es wird ein breiter exothermer Peak registriert. Beim 2. Heizlauf ist dieser deutlich schwächer ausgebildet. So kann in der 2. Heizkurve die $T_{g}$-Stufe besser quantifiziert werden.

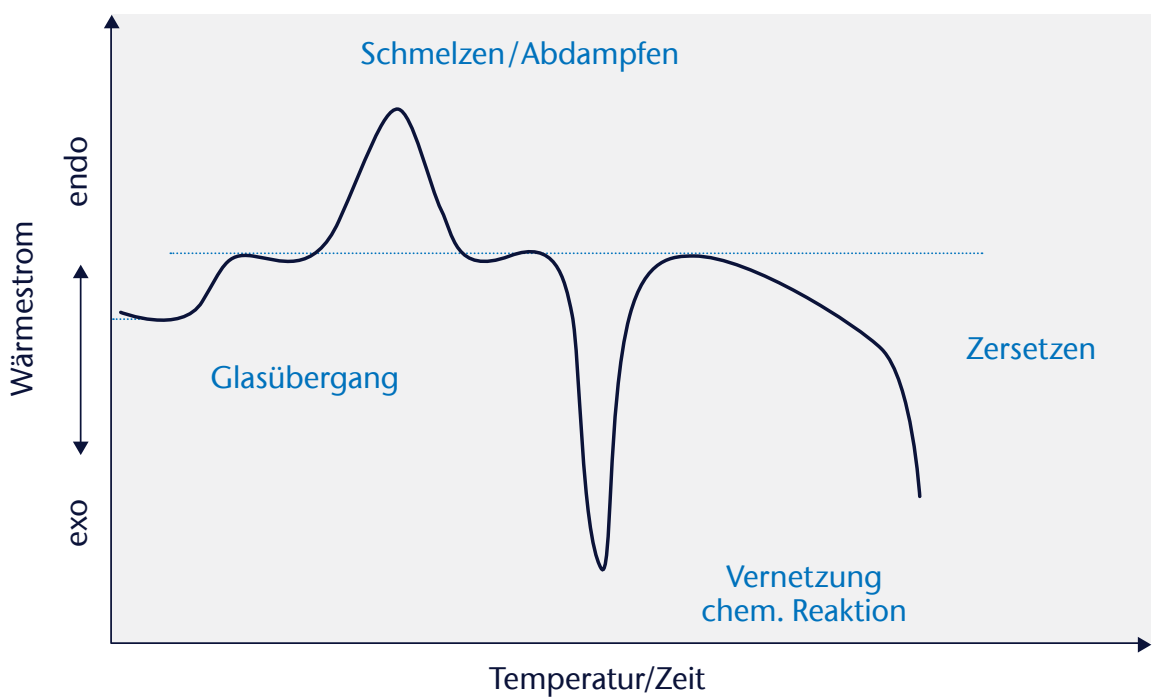

Abb. 3) DSC-Messkurve eines partiell kristallinen reaktiven Kunststoffs (schematisiert)

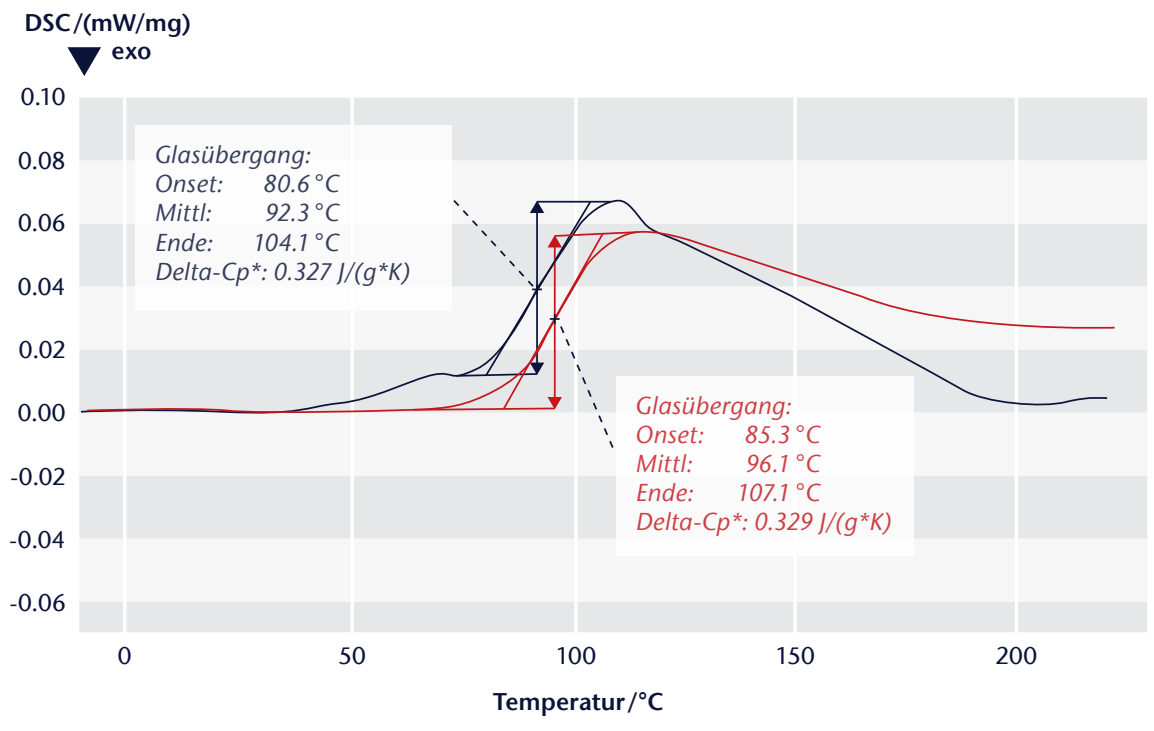

Abb. 4) DSC-Kurven eines Modell-PU, Schwarz - 1.Heizlauf, Rot - 2. Heizlauf 
3.1 PU-Systeme mit Sojaöl

In Abbildung 5 sind die DSC-Kurven (1. Heizlauf) für die Proben, die mit unterschiedlichen Anteilen von Sojaöl synthetisiert wurden, zusammengestellt.

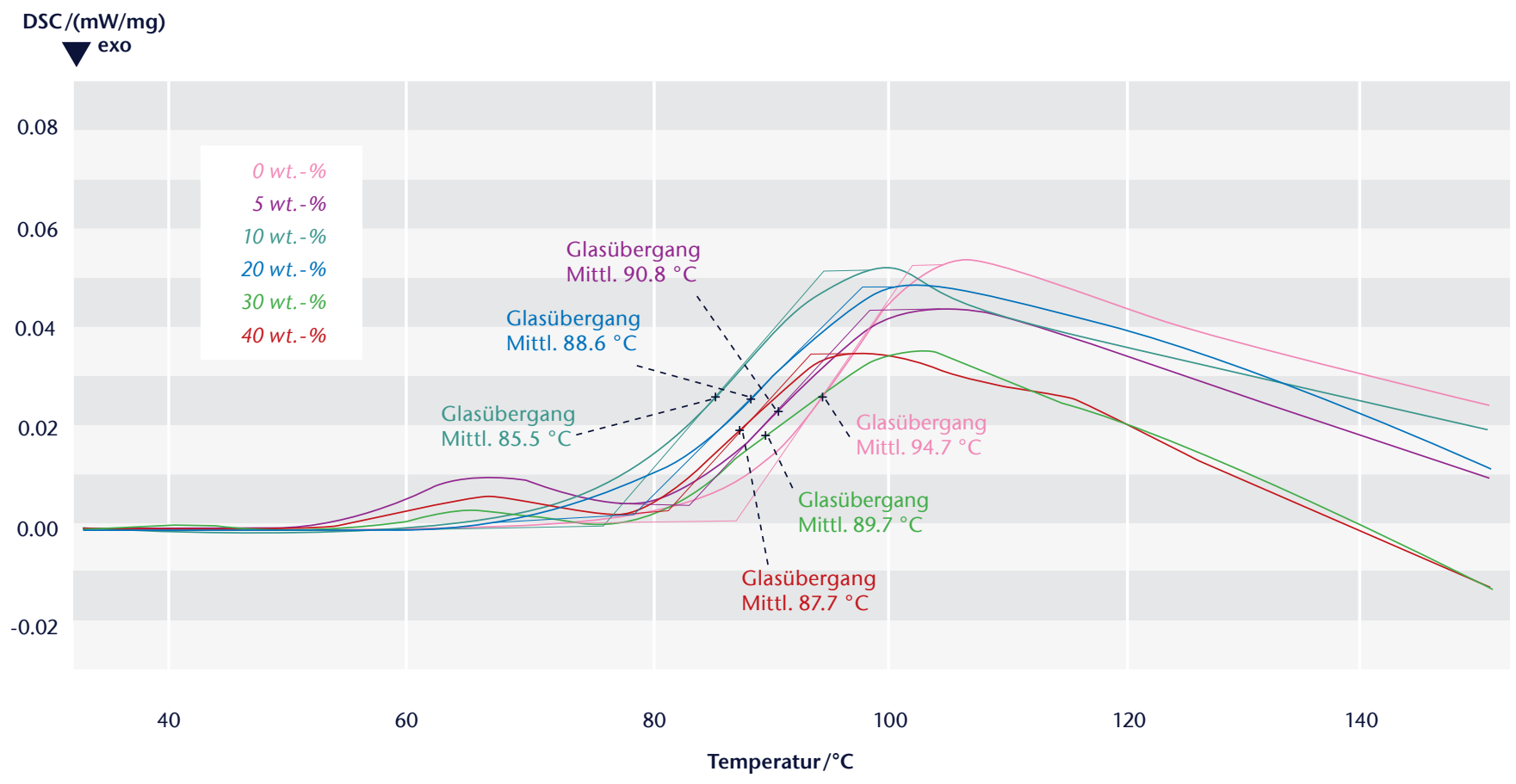

Abb. 5) DSC-Kurven der Proben mit 0, 5, 10, 20, 30 und 40 wt.-\% Sojaöl - 1. Heizlauf

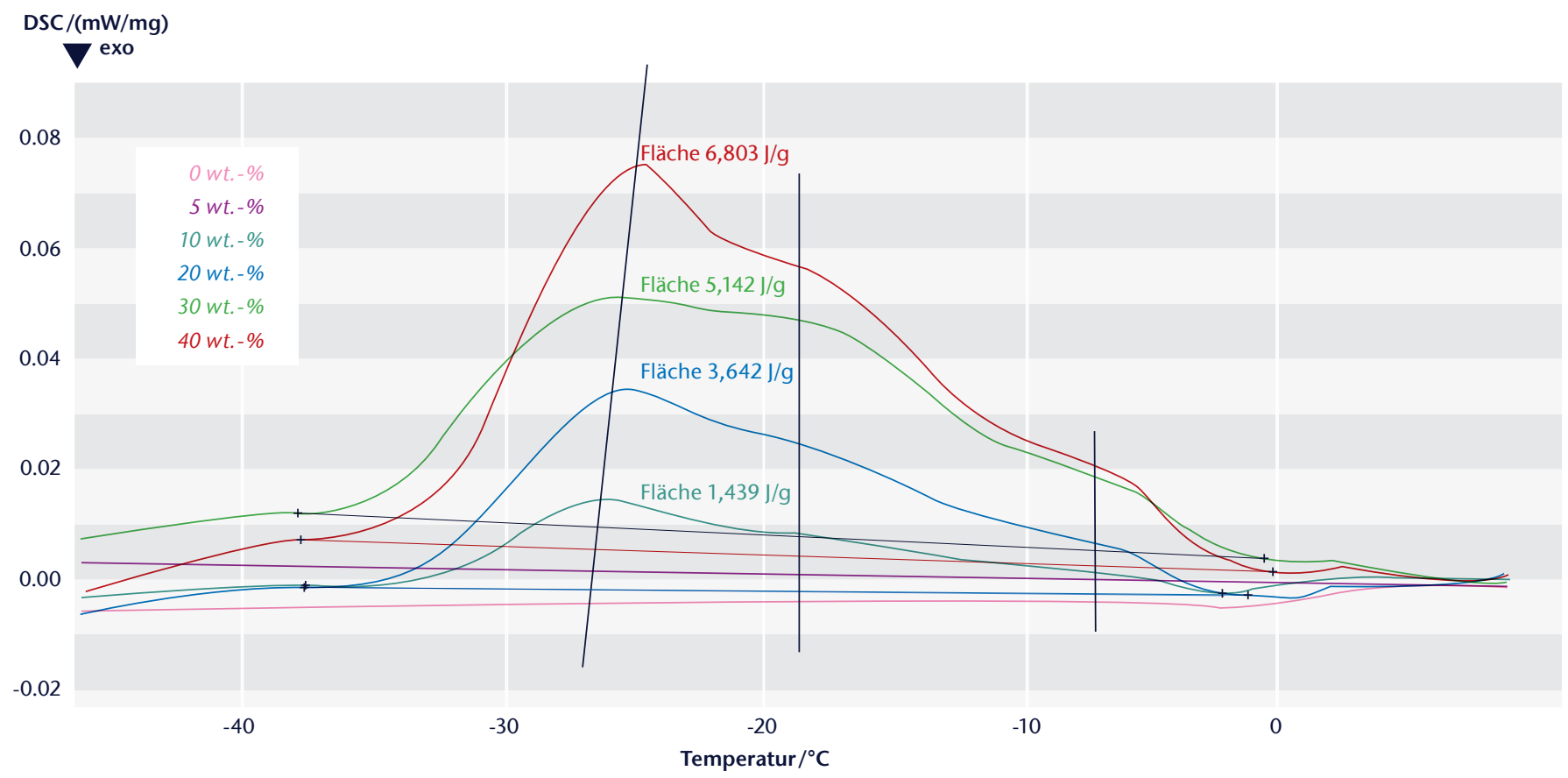

Abb. 6) DSC-Kurven der Proben mit 0, 5, 10, 20, 30 und 40 wt.-\% Sojaöl - 1. Heizlauf 
In Abbildung 6 ist der Ausschnitt der DSC-Kurven im Temperaturbereich von $-45^{\circ} \mathrm{C}$ bis etwa $10^{\circ} \mathrm{C}$ dargestellt. Hier sind die endothermen Effekte, die durch das Schmelzen einer kristallinen Phase entstehen, zu erkennen. Die Schmelzenthalpie $\Delta \mathrm{H}$ wird durch Integration des endothermen Peaks bestimmt (Höhne et al. 2003). Sie ist u. a. ein Maß für die Menge des kristallisationsfähigen Anteils in der Substanz. Wie der Abbildung 6 weiter zu entnehmen ist, besteht der Schmelzpeak aus der Überlagerung von mindestens drei Einzelprozessen. Diese sind durch drei Fraktionen unterschiedlicher Molmasse oder leicht variierter Kettenstruktur zu erklären. Die Lage des Einzelpeaks bei Temperaturen um $25^{\circ} \mathrm{C}$ wird mit höherem kristallinen Anteil zu höheren Temperaturen verschoben (durch Perfektion und/oder Größe der kristallinen Bereiche).

Abbildung 7 zeigt den Zusammenhang zwischen dem Sojaöl-Anteil und den gemessenen Werten für $\mathrm{T}_{\mathrm{g}}$ und $\Delta \mathrm{H}$. Wie der Abbildung 7 zu entnehmen ist, ergibt sich keine monotone Abhängigkeit von $\mathrm{T}_{\mathrm{g}}$ vom Anteil an Sojaöl. Die Schmelzenthalpie $\Delta \mathrm{H}$ im Bereich von ca. $-35^{\circ} \mathrm{C}$ und $0^{\circ} \mathrm{C}$ zeigt in Abhängigkeit vom Anteil an Sojaöl in grober Näherung einen linearen Zusammenhang. Der Wert für
5 wt.-\% Sojaöl bleibt etwa $\Delta \mathrm{H}=0 \mathrm{~J} / \mathrm{g}$, d. h. es ist noch kein Schmelzprozess zu beobachten. Ein geringer Anteil von weniger als 5 wt.-\% Sojaöl zeigt keine Kristallisation in einer eigenen separaten Phase. Vielmehr wird dieser Anteil wahrscheinlich molekular gelöst. Höhere Anteile bilden dann eine separate Phase aus, die eine Kristallisation des Sojaöls im PU-System zulässt.

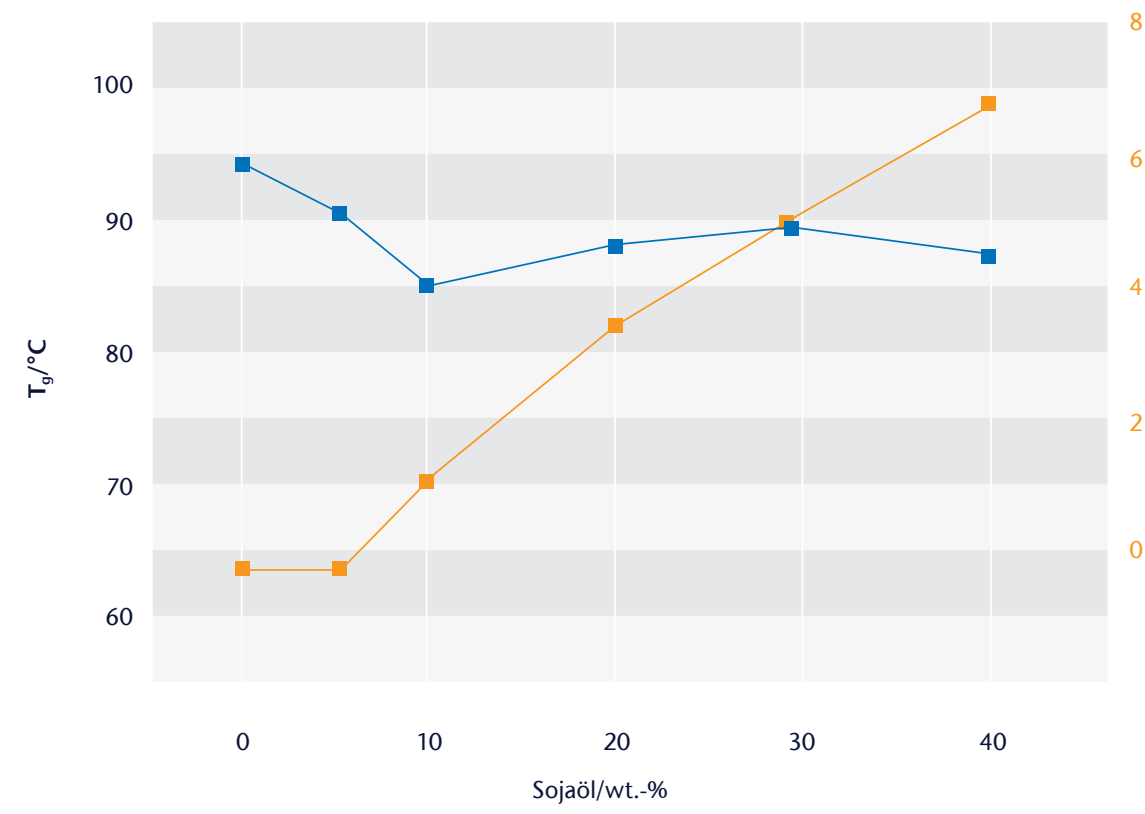

Abb. 7) $T_{g}$ und $\Delta H$ in Abhängigkeit vom Sojaölanteil, 1. Heizkurve
Diesen Schluss lässt auch der $\mathrm{T}_{\mathrm{g}}$-Verlauf in Abbildung $7 \mathrm{zu}$. $\mathrm{T}_{\mathrm{g}}$ wird bis etwa 10 wt.-\% Sojaöl durch dessen Weichmacherwirkung im molekular verteilten Zustand erniedrigt. Eine weitere Zugabe von Sojaöl bewirkt keine weitere Erniedrigung von $T_{g}$. $T_{g}$ bleibt nahezu konstant, da weiteres Sojaöl nicht mehr gemischt werden kann und deshalb eine disperse Phase bildet.

\subsection{PU-Systeme mit Rapsöl}

In Abbildung 8 ist der Ausschnitt der DSC-Kurven im Temperaturbereich von $-50^{\circ} \mathrm{C}$ bis etwa $10^{\circ} \mathrm{C}$ dargestellt. Auch hier sind endotherme Effekte, die durch das Schmelzen einer kristallinen Phase entstehen, zu erkennen.

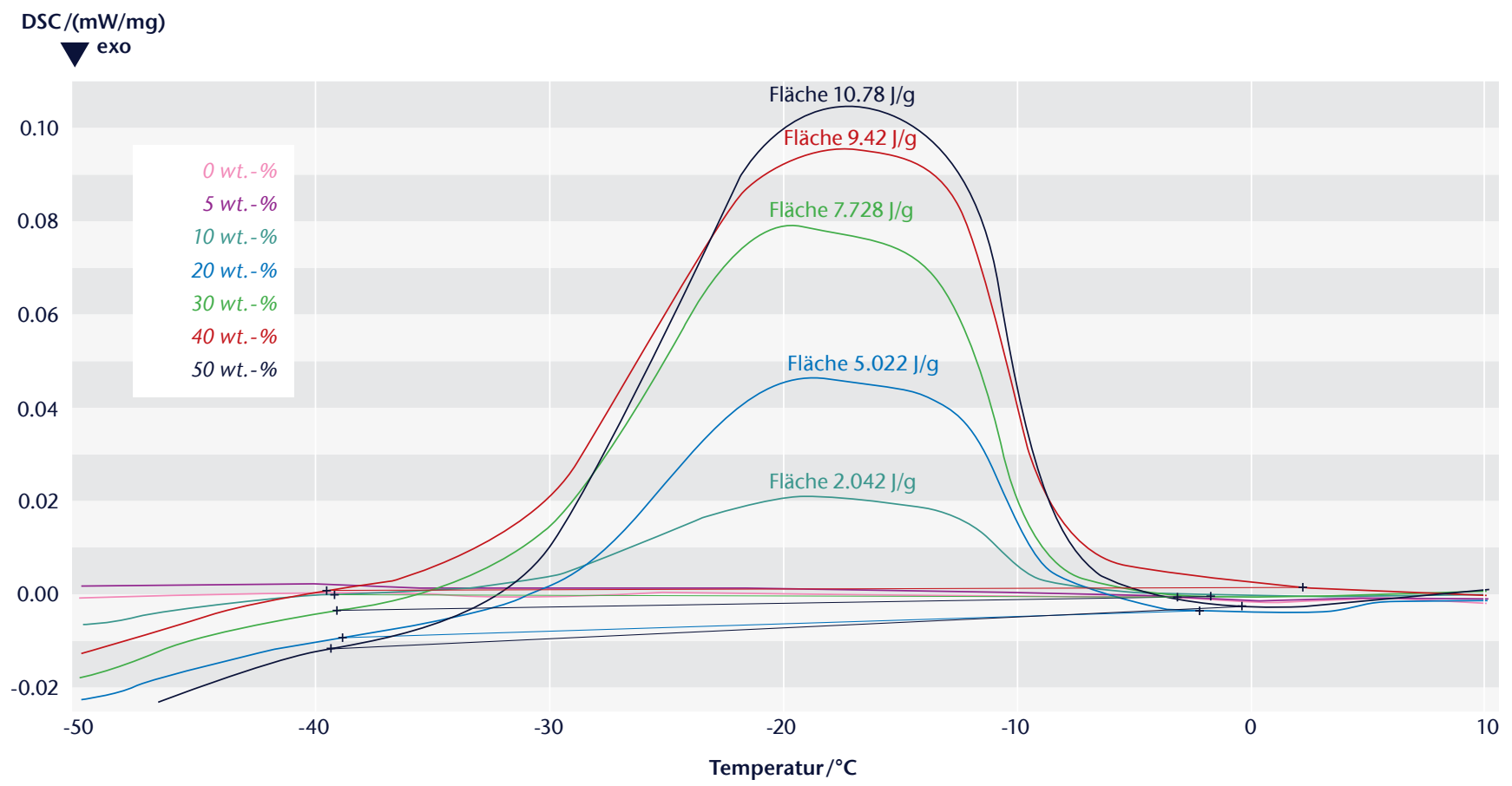


Der Abbildung 8 ist zu entnehmen, dass der Schmelzpeak aus der Überlagerung von mindestens zwei Einzelprozessen besteht. Wahrscheinlich auch hier durch Fraktionen unterschiedlicher Molmasse oder leicht variierter Kettenstruktur verursacht. Die Lage des Einzelpeaks bei Temperaturen um $-20^{\circ} \mathrm{C}$ wird mit höherem kristallinen Anteil um etwa $3 \mathrm{~K}$ zu höheren Temperaturen verschoben (durch Perfektion und/oder Größe der kristallinen Bereiche), während die Lage des Peaks um $-14^{\circ} \mathrm{C}$ in Abhängigkeit vom Rapsölanteil nahezu konstant bleibt.

Die den DSC-Kurven (1. Heizlauf) für die Proben, die mit unterschiedlichen Anteilen von Rapsöl synthetisiert wurden, entnommenen Werte für $T_{g}$ sowie $\Delta \mathrm{H}$ sind in der Abbildung 9 zusammengestellt. Wie der Abbildung $9 \mathrm{zu}$ entnehmen ist, ergibt sich für $T_{g}$ sowie $\Delta \mathrm{H}$ keine monotone Abhängigkeit vom Anteil an Rapsöl. Die in Abbildung 9 aufgetragene Schmelzenthalpie $\Delta \mathrm{H}$ im Bereich von ca. $35^{\circ} \mathrm{C}$ und $5^{\circ} \mathrm{C}$ zeigt in Abhängigkeit vom Anteil an Rapsöl, dass erst bei einem Anteil von mehr als 5 wt.-\% bis 10 wt.-\% Rapsöl eine kristalline Phase gebildet wird. Bei höheren Konzentrationen an Rapsöl steigt dann die Kristallinität weniger als proportional mit dem Rapsölanteil. Dieses Verhalten von $\mathrm{T}_{\mathrm{g}}$ und $\Delta \mathrm{H}$ hat die gleichen Ursachen, die bereits bei dem System mit Sojaöl diskutiert worden sind. Gemeinsam für beide PU-Systeme ist die Tatsache, dass bei mehr als 5 wt.-\% Soja- bzw. Rapsöl eine disperse Öl-Phase gebildet wird.

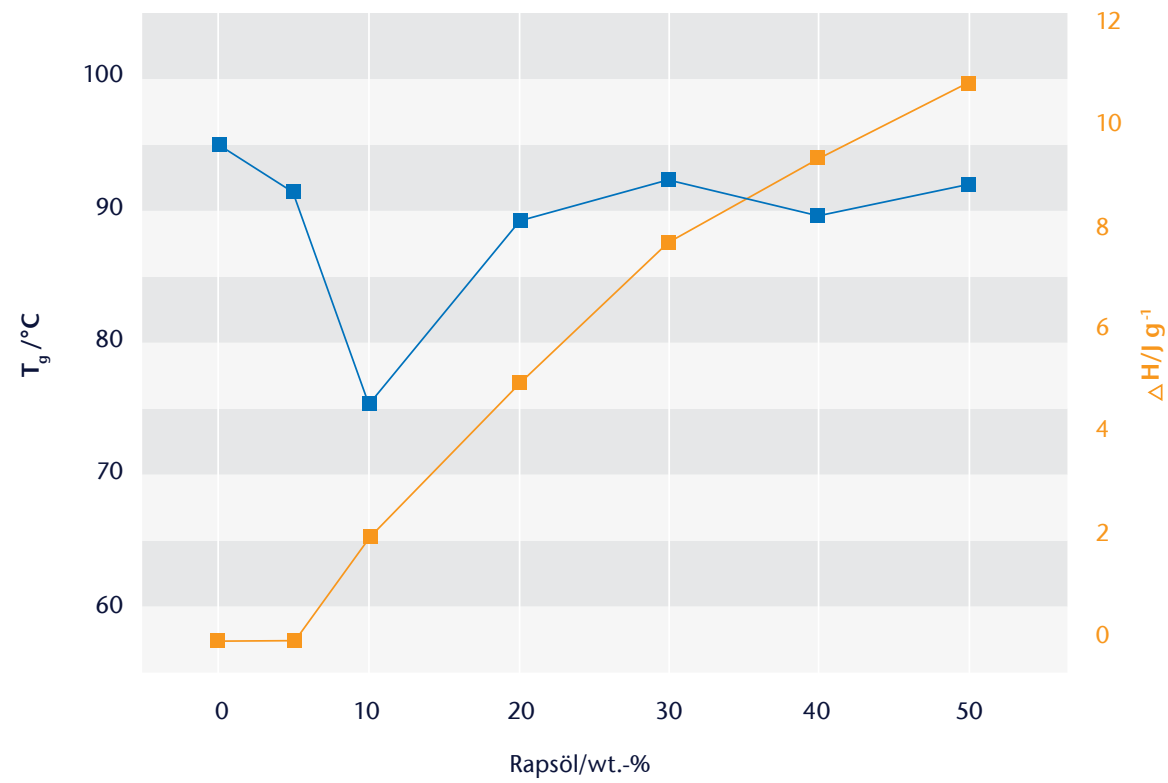

Abb. 9) $T_{g}$ und $\Delta H$ in Abhängigkeit vom Rapsölanteil, 1. Heizkurve

Für die detaillierte Strukturcharakterisierung muss eine Reihe zusätzlicher Informationen durch thermoanalytische und spektroskopische Messmethoden gewonnen werden. Neben diesen indirekten Methoden belegen abbildende Verfahren den direkten Nachweis für die diskutierte Phasenstruktur der PU-Öl-Systemen. Als Beispiel sind in Abbildung 10 die AFM Bilder des reinen PU-Systems und des mit 20 wt.-\% Rapsöl (bezogen auf das Diol) modifizierte PU zu sehen. Sehr anschaulich ist die Phasenseparation des Pflanzenöls durch die dunklen „Löcher“ von ca. $1 \mu \mathrm{m}$ in der Abbildung qualitativ zu erkennen. Die Löcher entstehen bei der Präparation der Messprobenoberfläche (Cryobruch).

\section{»IV. FAZIT}

Die beschriebenen neuartigen PUSysteme auf der Basis neuartiger hydrophober Diole und einem hohen Anteil nachwachsender Rohstoffe in Form von pflanzlichen Ölen zeigen eine spezifisch ausgebildete Phasenstruktur, die mithilfe thermischer und mikroskopischer Methoden bewiesen wurde. Die entwickelten PU-Systeme eignen sich sowohl als Vergussmassen als auch zum Laminieren. Mit vorgefertigten Folien, wie auch mit Pulver, können in Zukunft langfaserverstärkte Komposite mit einem hohen Niveau der mechanischen Eigenschaften erreicht werden.
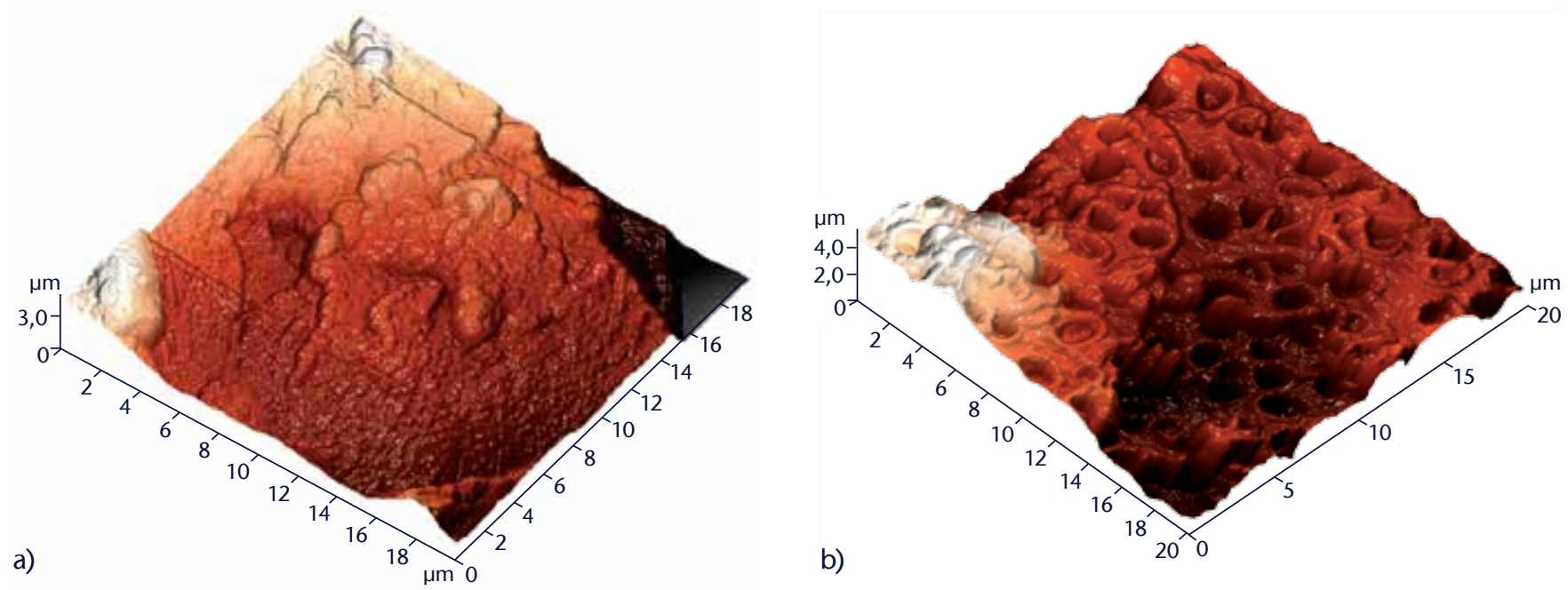

Abb. 10) AFM Visualisierung der Oberflächentopographie von PU-System ohne (a) und mit 20 wt.-\% Rapsöl im Diol (b) 


\section{LITERATUR}

Desroches, M., Escouvois, M., Auvergne, R., Caillol, S., Boutevin, B. (2012): From Vegetable Oils to Polyurethanes: Synthetic Routes to Polyols and Main Industrial Products, Polymer Reviews 52.

Ehrenstein, G. W., Riedel, G., Trawiel P. (2003): Praxis der Thermischen Analyse von Kunststoffen, 2. Auflage, Caral Hanser Verlag.

Hatakeyama, T. (1999): Fundamentals and Applications to Polymer Science, Second Edition, Wiley Verlag.

Höhne, G., Hemminger, W. F., Flammersheim, H.-J. (2003) Differential Scanning Calorimetry, Springer Verlag.

Ionescu, M. (2005): Chemistry and Technology of Polyols for Polyurethanes, Rapra Technology Limited.

Leppekes, R. (2003): Polyurethane: Werkstoff mit vielen Gesichtern (Elastogran), Die Bibliothek der Technik, Bd. 91 5., überarb. Auflage.

Menczel J.D., Prime R.B. (2009): Thermal analysis of polymers: Fundamentals and applications, John Wiley Verlag.

Oertel G. (1993): Polyurethane Handbook, 2nd ed.; Carl Hanser Verlag.

Randall, D., Lee, S. (2002): The Polyurethane Books, Huntsman International LIC.

Stoycheva, V., Paulmann, U. (2010): Neue Vergussmassen und deren Verwendung zur Herstellung von Elektroisolierstoffen, EP 2361940 A1 19.02.2010.

Stoycheva, V., Langenstraßen, R., Boyadzhiev, M., Behrendt, G., Paulmann, U. (2008): Neue PolyurethanVergussmassen und Verfahren zu ihrer Herstellung, DE 102008054940 A1, 18.12.2008.

\section{AUTOREN}

Dipl.-Ing. Vesela Stoycheva

University of Chemical Technology and Metallurgy

$8 \mathrm{KI}$. Ohridski bul.

1756 Sofia, Bulgaria

Telefon: +359 (0) 26254 / 101

vestoych@th-wildau.de

\section{Dr. Harald Goering}

Fachbereich 3.4, Sicherheit von Lagerbehältern

Bundesanstalt für Materialforschung und -prüfung

Unter den Eichen 87

12205 Berlin

Telefon: +49 (0) 30 / 81043309

harald.goering@bam.de

\section{Dipl-Chemikerin Uta Knoll}

Fachbereich Ingenieurwesen/Wirtschaftsingenieurwese

TH Wildau [FH]

Telefon: +49 (0) 3375 / 508459

uta.knoll@th-wildau.de 